\title{
Evaluación del comportamiento de los indicadores de productividad y rentabilidad financiera del sector petróleo y gas en Colombia mediante el análisis discriminante
}

\begin{abstract}
Efraín Javier de la Hoz Granadillo

Facultad de Ciencias Económicas, Universidad de Cartagena edelahozg@unicartagena.edu.co
\end{abstract}

\section{Tomás José Fontalvo}

\section{Herrera}

Facultad de Ciencias Económicas, Universidad de Cartagena. tfontalvoh@unicartagena.edu.co

José Morelos Gómez Facultad de Ciencias Económicas, Universidad de Cartagena jmorelosg@unicartagena.edu.co

\section{Resumen}

Este artículo presenta los resultados del comportamiento de los indicadores de productividad y rentabilidad del sector petróleo y gas en Colombia entre los años 2008 y 2010, el cual se realizó por medio de análisis discriminante. Inicialmente, se realizó un análisis del sector y una revisión teórica de la evaluación de la gestión financiera, del apalancamiento estratégico, de los stakeholders (partes interesadas), de la teoría de la agencia, de los indicadores de productividad y rentabilidad, así como de conceptos básicos de análisis discriminante. En la metodología utilizada se calcularon los indicadores de productividad y rentabilidad de 116 empresas del sector petróleo y gas a partir de los estados financieros presentados en los periodos de estudio. Seguidamente, se utilizó la técnica de análisis discriminante para explicar la pertenencia y discriminación de los indicadores estudiados. De la función discriminante y medios obtenidos se puede concluir que los indicadores analizados no presentan diferencias importantes, lo que muestra un periodo de estancamiento en los periodos estudiados. Con el trabajo de investigación se pudo concluir que existe una diferencia significativa sólo para el indicador margen bruto. El modelo de la función discriminante permite una efectividad de clasificación de $57.3 \%$.

Palabras clave: productividad, rentabilidad, indicadores financieros, gestión empresarial, gestión financiera 


\title{
Assessment of the behavior of productivity and financial profitability indicators in the oil and gas sector in Colombia through discriminant analysis
}

\begin{abstract}
This research paper presents the results of the behavior of productivity and profitability indicators in the oil and gas sector in Colombia in the years 2008 and 2010 by means of discriminant analysis. Initially there is an analysis of the sector and a theoretical review of the financial management assessment, strategic leverage, stakeholders, agency theory, productivity and profitability indicators and basic concepts of discriminant analysis. In the methodology used, productivity and profitability indicators were calculated for $116 \mathrm{com}$ panies in the oil and gas sector from the financial statements in the periods of study. Then, the discriminant analysis technique was used to explain the belonging and discrimination of the indicators studied. From the discriminant function and the means obtained it can be concluded that the analyzed indicators do not present significant differences, which shows a period of stagnation in the periods studied. With the research work it was concluded, by discriminant analysis, that there is a significant difference just for the indicator gross margin. The discriminant function model allows an effective classification of $57.3 \%$.
\end{abstract}

Keywords: productivity, profitability, financial indicators, business management, financial management

\section{Introducción}

Esta investigación busca analizar el comportamiento de los indicadores de productividad y rentabilidad financiera en el sector petróleo y gas en Colombia para que se puedan tener criterios claros, tomar decisiones y establecer si existe mejoramiento en los indicadores estudiados. Esto permitirá estudiar las variables, los rubros e indicadores que generan causalidad en el mejoramiento de la gestión del sector para decidir las actividades que impacten en los grupos de interés.

La globalización de los mercados ha convertido la gestión empresarial en una tarea competitiva para la supervivencia de la empresa, dado el creciente nivel de incertidumbre al que se enfrentan los directivos en el proceso de toma decisiones (Vázquez, 2001). En esta línea, las organizaciones deben adoptar metodologías que incorporen análisis estadísticos de resultados conducentes a mejorar la gestión disminuyendo los niveles de riesgo y apoyando las decisiones gerenciales. En Colombia el sector petrolero ha venido cobrando relevancia en su participación den- 
tro del escenario internacional, pues es uno de los seis países latinoamericanos que posee recursos energéticos significativos y potenciales hacia el futuro. Se estima que sus reservas probadas ascienden a los 1.500 billones de barriles de petróleo y 7.3 trillones de pies cúbicos en gas natural; ese petróleo representa el $26 \%$ del total de las exportaciones colombianas, constituyéndose en una de las mayores fuentes externas de efectivo para el país (ANH, 2007).

En este artículo se desarrolló una metodología para el análisis sistemático de los indicadores de productividad y rentabilidad de las empresas del sector petróleo y gas en Colombia, quienes presentaron informes de sus estados financieros en organismos como la Superintendencia de Sociedades y las Cámaras de Comercio correspondientes. En la evaluación de los indicadores señalados se utilizó el análisis discriminante a partir de razones financieras en las que se definieron las variables dependientes e independientes en el modelo de función discriminante, de manera que se minimiza la varianza dentro de los grupos y se maximiza la varianza entre grupos; para ello fue necesario la verificación de supuestos de normalidad e igualdad de matrices varianza-covarianza de las variables objeto de estudio, que permitieron analizar diferencias significativas y la evolución en los indicadores seleccionados.

En concordancia con lo anterior, para el análisis se utilizaron los indicadores de productividad y rentabilidad; evaluando el desempeño de las compañías del sector, de tal forma que se pudo realizar un análisis comparativo de los resultados de productividad y rentabilidad en las empresas del sector petróleo y gas en Colombia entre 2008 y 2010. Los resultados indican que no existe un mejoramiento significativo de los indicadores estudiados, con lo cual se demuestra la falta de una gestión corporativa y de una política estatal que dinamice el sector. Es importante resaltar que la investigación presenta una metodología de análisis robusta para la evaluación de indicadores financieros, la cual puede ser extendida en su aplicación a diferentes sectores empresariales siempre y cuando se tengan en cuenta las particularidades de cada sector.

\section{Referencial teórico}

Para el desarrollo de este artículo de investigación se trabajó con los referentes teóricos y conceptuales asociados con el sector energético, subsector petróleo y gas en Colombia, así como el apalancamiento estratégico, la evaluación de la gestión financiera, indicadores de productividad y rentabilidad, conceptos básicos de 
análisis discriminante, lo relacionado con los criterios para el análisis de la productividad y rentabilidad del sector petrolero y gas en Colombia.

\section{Sector energético}

El sector energético es una variable que impulsa el nivel de vida de las sociedades, pero también puede convertirse en un factor de conflictos cuando no se gestiona bien (Altahyde, 2012). Ahora bien, el petróleo es sin lugar a dudas el principal energético del mundo dado sus condiciones como combustible, lo que proporciona poder económico en los países productores llegando inclusive en algunas economías a establecer gran parte de sus ingresos en este rubro. Asimismo, a diferencia del carbón, este recurso se encuentra mayoritariamente en países subdesarrollados, otorgando un potencial de desarrollo en el escenario mundial (Rodríguez, 2004).

Según Selling y Stickney (1989), los conceptos microeconómicos y las estrategias de negocios son importantes para comprender el comportamiento en el tiempo de indicadores financieros como la tasa de retorno sobre activos (ROA, return on assets), los cuales se pueden utilizar para generar interpretaciones muy acertadas sobre las tendencias y las diferencias entre las empresas y las industrias.

En este sentido, Giner (1990) plantea que la disposición de información contable adecuada para la toma de decisiones puede tener importantes consecuencias en los resultados económicos de las organizaciones. Por su parte, Stickney (1990) anota que las compañías del sector energético caracterizadas por altas inversiones de capital y elevados costos fijos requieren operar con elevados márgenes que aseguren la rentabilidad del capital y faciliten la consecución de fuentes de financiación.

En cuanto al apalancamiento estratégico de una empresa, éste proporciona flexibilidad para modificar su posición relativa en el mercado en relación con los competidores generando cambios en los ingresos o en la participación en el mercado (Milind, 1995). Definitivamente el estudio de los apalancamientos permite que la empresa tenga entre sus manos una poderosa herramienta de análisis y apoyo en la toma de decisiones estructurales de la misma.

\section{El sector petróleo y gas en Colombia}

El gobierno colombiano se ha visto interesado en impulsar el sector petrolero a través de la búsqueda de maneras para aumentar la producción, por lo que ha em- 
prendido estrategias para la atracción de inversiones importantes provenientes del resto del mundo, convirtiéndose el sector en uno de los más importantes de Latinoamérica, según el Ministerio de Relaciones Exteriores (2011).

Sin duda, el petróleo es uno de los recursos naturales más importantes en la economía de cualquier país; es decir, el bien básico que más se corrompe en el mundo (López et al., 2012). A esta característica se suma el hecho de ser un bien superior, condición que proviene no solamente de los aspectos no competitivos de dicho mercado, sino también del hecho de ser un recurso natural no renovable.

De esta manera, la importancia del petróleo en el país no es una tendencia que se ha dado desde siempre debido a que la participación del sector petrolero en la balanza comercial ha tenido un comportamiento de alzas y bajas en las exportaciones. Según cifras del Departamento Nacional de Estadísticas, se muestra que en el 2008 las exportaciones de petróleo y sus derivados pasaron de 12212578 miles de dólares FOB en el 2008 a 10267502 miles de dólares FOB en el 2009, subiendo a 16501625 miles de dólares FOB en el 2010 (DANE, 2011).

Por su parte, Villamil (2007) asegura que en 1999 hubo una bonanza petrolera cuando justamente los campos de Cusiana y Cupiagua fueron la base de la economía petrolera del país, llegando a aportar más de la mitad de la explotación nacional con una cifra récord de 430 mil barriles diarios de crudo. En su conjunto, los campos operados por la British Petroleum y Occidental sumaron en 1999 cerca del $80 \%$ de la producción petrolera colombiana.

Asimismo, de 2004 a 2012 las exportaciones han tenido una tendencia de aumento, salvo de 2008 a 2009 que hubo una disminución de 1945076 miles de dólares FOB, registrándose el aumento más significativo en estas exportaciones de 2010 (16 485082 miles de dólares FOB) a 2011 (27 953826 miles de dólares FOB).

De igual manera, el comportamiento de la producción del crudo posiciona al sector petrolero como uno de los más importantes sectores de Colombia, así como uno de los sectores petroleros más importantes de Latinoamérica, según La British Petroleum es el tercer país en la región en producción de petróleo (Ministerio de Relaciones Exteriores, 2011).

La participación en el PIB de hidrocarburos creció $17.5 \%$ en 2011, valor que se compara con el 19.2\% registrado el año anterior. Así, este sector promedia una tasa de crecimiento de $16.7 \%$ en los últimos cuatro años. En términos de la inversión 
extranjera directa, la dirigida al petróleo y la minería representa cerca del 85\% del total. Sólo los planes de inversión de las principales compañías suman cerca de USD 10000 millones para 2012. El área de Investigaciones Económicas de Correval (2012) señala que en 2011 se perforaron 125 pozos exploratorios y la meta del Gobierno para 2012 era de 178.

\section{Evaluación de la gestión financiera en el sector petróleo y gas en Colombia}

Esta investigación se basó en un conjunto de conocimientos teóricos soportados en un análisis discriminante multivariado (ADM) que permitieron analizar el impacto y afectación de la gestión financiera en las empresas del sector petrolero en Colombia.

Fontalvo et al. (2012a) indican que la evaluación de indicadores como técnica administrativa ayuda a mejorar la gestión porque produce la información necesaria para identificar y entender las causas de los logros y los fracasos o problemas del desempeño individual y colectivo dentro de un contexto de planeación estratégica. Esta evaluación saca a la luz información real sobre el comportamiento del sistema objeto de estudio, que hace posible la creación de estrategias y herramientas de apoyo para la toma de decisiones que contribuyan a mejorar la estructura del mismo. Nociones como éstas son las que buscan integrar esta investigación, así como el considerar la metodología y las técnicas utilizadas, tomando en cuenta que la gestión financiera se evalúa en cómo estos conceptos afectan la rentabilidad y productividad del sector.

Por otra parte, Navas y Marbelís (2009) señalan que una empresa debe implementar medidas que le permitan ser más competitiva y eficiente desde la perspectiva económica y financiera; de forma tal que haga mejor uso de sus recursos para obtener mayor productividad y mejores resultados con menores costos, lo que implica la necesidad de realizar un análisis exhaustivo de la situación económica y financiera de la actividad que lleva a cabo. Al respecto, Díaz (2009) afirma que una forma más sencilla de realizar este análisis es mediante la formulación de indicadores financieros que permitan establecer relaciones entre dos o más elementos de los estados contables, jugando un papel importante en el proceso de comercialización y los ingresos percibidos.

Por otro lado, Palacio (2008) asegura que el diagnóstico correctamente realizado permite identificar en tiempo y forma los posibles problemas económicos y finan- 
cieros de la empresa, identificar sus causas y, lo que es más importante, establecer medidas correctoras con tiempo suficiente para que actúen. Nuñez y Vieites (2009) señalan que el diagnóstico de la gestión financiera permitirá a la empresa atender correctamente sus compromisos financieros, financiar adecuadamente las inversiones, así como mejorar ventas y beneficios, aumentando el valor de la empresa.

De esta manera, en este artículo se analizan los indicadores de productividad y rentabilidad de las organizaciones, objeto de estudio, para determinar si existen cambios significativos del sector en los años 2008 y 2010.

\section{Indicadores de productividad}

El Banco Nacional de Comercio Exterior de México (1991) expresa la productividad como el cambio cualitativo que proporciona mejores formas de hacer las cosas, así como la utilización racional de los recursos, la aplicación de la innovación y los avances tecnológicos con la participación de la población en la actividad económica y sus resultados. Berechet y San Miguel (2006) señalan que los indicadores de productividad permiten fijar una relación entre la cantidad de bienes y servicios producidos y la cantidad de recursos utilizados, transformándose en un factor crucial para la determinación del eficiente uso de los recursos. Levy (2004) señala que los indicadores de productividad son los que muestran la eficiencia en la operación de la empresa, íntimamente relacionados con los resultados del negocio.

En consecuencia, los indicadores de productividad representan un elemento clave para la creación de riquezas dentro de una empresa por permitir la realización de inversiones en mejores recursos productivos como nuevas tecnologías; esta situación se traduce en una ventaja competitiva e incremento de los sueldos, lo que acrecentará el volumen de la demanda agregada, que resulta de la dinamización de la economía.

Por otro lado, la medición de la productividad es un procedimiento necesario para el desarrollo y la proyección de las actividades económicas de cualquier organización; éste se lleva a cabo mediante la aplicación de indicadores que relacionan diversas variables. Desempeñando así un papel esencial en la evaluación de la producción porque pueden definir no solamente el estado actual de los procesos, sino que, además, son útiles para proyectar el futuro de los mismos. 
Dentro de una organización los indicadores de productividad se pueden medir con respecto a un factor de producción determinado, motivo por el cual existe la posibilidad de que se presente una amplia gama de indicadores referentes a diferentes áreas; aunque los más importantes, según Miranda y Toirac (2010), son los relacionados con la productividad del trabajo, la productividad del uso de los materiales y la productividad del capital. En el cuadro 1 se presentan las ecuaciones ( 1 a la 6) donde se muestra cómo se calcularon los indicadores de productividad para esta investigación.

\section{Cuadro 1 \\ Indicadores de productividad}

\begin{tabular}{|c|c|c|}
\hline Indicador & Ecuación & \\
\hline \multirow{2}{*}{ IP1 } & Utillidad Bruta & \multirow{2}{*}{$\mathrm{x} 100(1)$} \\
\hline & Valor agregado (ventas-pagosaproveedores+inventarios) & \\
\hline \multirow{2}{*}{ IP2 } & Utillidad Operacional & \multirow{2}{*}{$\mathrm{x} 100(2)$} \\
\hline & Valor agregado (ventas-pagos a proveedores+inventarios) & \\
\hline \multirow{2}{*}{ IP3 } & Utillidad Neta & \multirow{2}{*}{$\mathrm{x} 100(3)$} \\
\hline & Valor agregado (ventas-pagos a proveedores+inventarios) & \\
\hline \multirow{2}{*}{ IP4 } & Valor agregado (ventas-pagos a proveedores+inventarios) & \multirow{2}{*}{$\mathrm{x} 100(4)$} \\
\hline & Capital Operativo (activos corrientes y fijo) & \\
\hline \multirow{2}{*}{ IP5 } & Utilidad Operacional & \multirow{2}{*}{ x 100(5) } \\
\hline & Capital Operativo (activos corrientes y fijo) & \\
\hline \multirow{2}{*}{ IP6 } & Utilidad Neta & \multirow{2}{*}{ x $100(6)$} \\
\hline & Capital Operativo (activos corrientes y fijo) & \\
\hline
\end{tabular}

\section{Indicadores de rentabilidad}

Ortiz (2011) señala que los indicadores de rentabilidad, denominados también de rendimiento o lucratividad, sirven para medir la efectividad de la administración de la empresa para controlar el costo y el gasto, y así convertir las ventas en utilidades. Asimismo, los indicadores referentes a rentabilidad tratan de evaluar la cantidad de utilidades obtenidas con respecto a la inversión que las originó, ya sea considerando en su cálculo el activo total o el capital contable (Guajardo, 2002).

De igual forma, Brealey y Myers (1998) aseguran que los analistas financieros utilizan índices de rentabilidad para evaluar qué eficientes son las empresas en el uso de sus activos. En el cuadro 2 se muestran las ecuaciones ( 7 a la 10) que representan los indicadores de rentabilidad utilizados en la presente investigación, así como la manera en que se calcularon. 


\section{Cuadro 2}

\section{Indicadores de rentabilidad}

\begin{tabular}{c|c}
\hline Indicador & Ecuación \\
\hline Margen bruto & $\frac{\text { Utillidad Bruta }}{\text { ingresos operacionales }} \times 100(7)$ \\
\hline $\begin{array}{c}\text { Margen } \\
\text { operacional }\end{array}$ & $\frac{\text { Utillidad operacional }}{\text { ingresos operacionales }} \times 100(8)$ \\
\hline Margen neto & $\frac{\text { ganancias y perdidas }}{\text { ingresos operacionales }} \times 100(9)$ \\
\hline Utilidad operacioal & margen de contribución $-\operatorname{costos~fijos~(10)~}$ \\
\hline
\end{tabular}

Análisis discriminante

El análisis discriminante es una técnica estadística multivariada de especial interés en la investigación, puesto que permite establecer la pertenencia probabilística de un individuo a grupos previamente establecidos a través de funciones de discriminación (Carvajal et al., 2004). Fundamentalmente, este análisis consiste en clasificar observaciones previamente obtenidas cuya clasificación tiene como punto de partida un conjunto de variables que caracterizan los individuos u objetos que se pretenden estudiar (Elizondo y Altman, 2003).

Igualmente, Vallejo (2012) asegura que el análisis discriminante es una técnica estadística que permite estudiar las referencias entre dos o más grupos de objetos con respecto a varias variables simultáneamente. Asimismo, uno de los objetivos básicos del análisis discriminante que presenta Vallejo es la identificación o caracterización de los grupos; asegura que esto puede servir para establecer diferencias entre ellos. En el caso de esta investigación se analizaría si existen diferencias entre dos periodos distintos de las empresas del sector petrolero y gas en Colombia, constituyendo estos dos periodos los grupos a los cuales se refiere Vallejo.

Por otra parte, Mures et al. (2005) sostienen que el objetivo de un análisis discriminante es analizar la relación entre una variable dependiente categórica con $g$ modalidades, que corresponden con los grupos analizados, y un conjunto de variables independientes métricas o cuantitativas a partir de una serie de funciones discriminantes. De este modo, las variables utilizadas en el análisis de la productividad y rentabilidad del sector petróleo y gas en Colombia son denominadas variables discriminantes. Éstas deben ser medidas en la escala de intervalo o razón para que 
las medias y varianzas puedan ser calculadas e interpretadas, modelo matemático que se presenta en las ecuaciones (18) y (19).

\section{Distancia de Mahalanobis}

Como criterio para seleccionar las variables que mejor discriminan en el análisis de la productividad y rentabilidad del sector petróleo y gas en Colombia, se utilizó la distancia D2 de Mahalanobis, la cual es una medida que se basa en la distancia euclidiana al cuadrado generalizada que se adecua a varianzas desiguales. La regla de selección en este procedimiento es maximizar la distancia D2 de Mahalanobis. La distancia multivalente entre los grupos cualesquiera $i$ y $j$ se define como ecuación (11):

$$
D_{i j}^{2}(X)=(n-m) \sum_{i=1}^{p} \sum_{j=1}^{p} H_{i j}\left(X_{i}^{(i)}-X_{i}^{(j)}\right)\left(X_{j}^{(i)}-X_{j}^{(j)}\right)
$$

Donde $n$ es el número de casos válidos, $\mathrm{k}$ es el número de grupos, $\mathrm{X}_{\mathrm{i}}^{(\mathrm{i})}$ es la media del grupo $i$ en la i-ésima variable independiente, $\mathrm{X}_{\mathrm{i}}^{(\mathrm{j})}$ es la media del grupo $j$ en la i-ésima variable independiente, $\mathrm{y}_{\mathrm{ij}}{ }^{-1}$ es un elemento de la inversa de la matriz de varianzas covarianzas intra-grupos. Siendo la variabilidad total de la forma, ecuación (12).

$$
G_{\mathrm{ij}}=\mathrm{H}_{\mathrm{ij}}+\mathrm{E}_{\mathrm{ij}}
$$

Donde, la covarianza total es igual a la covarianza dentro de grupos más la covarianza entre grupos.

Así, la probabilidad de que un objeto j, con una puntuación discriminante $\mathrm{D}=\left(\mathrm{y}_{\mathrm{j} 1}, \ldots, \mathrm{y}_{\mathrm{jm}}\right)$ pertenezca al grupo i-ésimo se puede estimar mediante la regla de Bayes, ecuación (13):

$$
\mathrm{P}\left(\mathrm{K}_{\mathrm{i}} / \mathrm{D}\right)=\frac{\mathrm{P}\left(\mathrm{D} / \mathrm{K}_{\mathrm{i}}\right) \mathrm{P}\left(\mathrm{K}_{\mathrm{i}}\right)}{\sum_{\mathrm{i}}^{\mathrm{m}} \mathrm{P}\left(\mathrm{D} / \mathrm{K}_{\mathrm{i}}\right) \mathrm{P}\left(\mathrm{K}_{\mathrm{i}}\right)}
$$

$\mathrm{P}\left(\mathrm{K}_{\mathrm{i}}\right)$ es la probabilidad a priori y es una estima de la confianza de que un objeto pertenezca a un grupo si no se tiene información previa.

Como cualquier otra técnica estadística, la aplicación de la misma ha de ir precedida de una comprobación de los supuestos asumidos por el modelo. El análisis discriminante se apoya en los siguientes supuestos: 
- Se tiene una variable categórica y el resto de variables son de intervalo o de razón y son independientes.

- Es necesario que existan al menos dos grupos; para cada uno de ellos se necesitan dos o más casos.

- El número de variables discriminantes debe ser menor que el número de objetos menos dos (2). $x_{1}, x_{2}, x_{3}, \ldots, x_{i}$, donde $\mathrm{i}<(\mathrm{n}-2)$ y n es el número de objetos.

- Ninguna variable discriminante puede ser combinación lineal de otras variables discriminantes.

- El número máximo de funciones discriminantes es igual al mínimo entre el número de variables y el número de grupos menos 1 (con q grupos, $(\mathrm{q}-1)$ funciones discriminantes).

- Las matrices de covarianzas dentro de cada grupo deben ser aproximadamente iguales.

- Las variables continuas deben seguir una distribución normal multivariante.

Regla de Bayes como criterio de clasificación

Las probabilidades a priori se definen de la siguiente forma,

$$
p_{i}=P\left(x \in \pi_{i}\right)
$$

En el caso donde el número de grupos a clasificar es dos (2) $i=2$, obtenemos las siguientes probabilidad a priori

$$
p_{1}=P\left(x \in \pi_{1}\right)
$$

Definida como la probabilidad a priori de pertenecer a la población uno (1).

Por otro lado, se tiene que:

$$
p_{2}=P\left(x \in \pi_{2}\right)
$$

la probabilidad a priori de pertenecer a la población dos (2). Una vez definidas estas probabilidades obtenemos las siguientes regiones de clasificación,

$$
R_{1}: \frac{f_{1}(x)}{f_{2}(x)} \geq\left[\frac{C(1 / 2)}{C\left({ }^{2} / 1\right)}\right]\left[\frac{p_{2}}{p_{1}}\right]
$$




$$
R_{2}: \frac{f_{1}(x)}{f_{2}(x)}<\left[\frac{C(1 / 2)}{C\left({ }^{2} / 1\right)}\right]\left[\frac{p_{2}}{p_{1}}\right]
$$

Generalmente se asume que $\left[\frac{C(1 / 2)}{C\left({ }^{2} / 1\right)}\right]=1$, como también $\left[\frac{p_{2}}{p_{1}}\right]=1$.

Formando las siguientes regiones de clasificación,

$$
\begin{gathered}
R_{1}: D(x)_{(x)} \geq 0 \\
R_{2}: D(x)_{x}<0
\end{gathered}
$$

Siendo la función discriminante

$$
D(x)=\ln \left[\frac{f_{1}(x)}{f_{2}(x)}\right]
$$

Método de clasificación

Existen básicamente tres métodos de clasificación para encausar los objetos o individuos a los $i$ grupos

- Método gráfico

En este caso se realiza la presentación del objeto o vector aleatorio $x_{0}$ en la nube de punto de los $i$ grupos y en forma visual determinar en qué grupo se ubica.

- Función discriminante

Desarrollando la función como, ecuación (18).

$$
D_{i j}^{2}(X)=(n-m) \sum_{i=1}^{p} \sum_{j=1}^{p} H_{i j}\left(X_{i}^{(i)}-X_{i}^{(j)}\right)\left(X_{j}^{(i)}-X_{j}^{(j)}\right)
$$


- Clasificación por probabilidad a posteriori

Se toma la probilidad de clasificar en $R_{i}$ un vector observado $x_{0}$ mediante la medida de probabilidad condicional de la forma,

$$
P\left(R_{i} / x_{0}\right)
$$

De donde se tiene que la probabilidad condicional, ecuación (19).

$$
P\left(R_{i} / x_{0}\right)=\frac{P\left(R_{i} \cap x_{0}\right)}{P\left(x_{0}\right)}=\frac{P\left(R_{i}\right) P\left(x_{0} / R_{i}\right)}{P\left(x_{0}\right)}=\frac{P_{i} f_{i}\left(x_{0}\right)}{P\left(x_{0}\right)}
$$

En dos poblaciones se tiene

$$
\begin{gathered}
P\left(R_{1} / x_{0}\right)=\frac{P\left(R_{1} \cap x_{0}\right)}{P\left(x_{0}\right)}=\frac{P\left(R_{1}\right) P\left(x_{0} / R_{1}\right)}{P\left(x_{0}\right)}=\frac{P_{1} f_{1}\left(x_{0}\right)}{P\left(x_{0}\right)} \\
P\left(R_{2} / x_{0}\right)=\frac{P\left(R_{2} \cap x_{0}\right)}{P\left(x_{0}\right)}=\frac{P\left(R_{2}\right) P\left(x_{0} / R_{2}\right)}{P\left(x_{0}\right)}=\frac{P_{2} f_{2}\left(x_{0}\right)}{P\left(x_{0}\right)}
\end{gathered}
$$

la probabilidad total, ecuación (20).

$$
P\left(x_{0}\right)=P\left(R_{1}\right) P\left(x_{0} / R_{1}\right)+P\left(R_{2}\right) P\left(x_{0} / R_{2}\right)=P_{1} f_{1}\left(x_{0}\right)+P_{2} f_{2}\left(x_{0}\right)
$$

Seleccionado las ecuaciones (19) y (20) se contruyen las siguientes probabilidades condicionales, ecuaciones (21) y (22).

$$
\begin{gathered}
P\left(R_{2} / x_{0}\right)=\frac{f_{2}\left(x_{0}\right)}{f_{1}\left(x_{0}\right)+f_{2}\left(x_{0}\right)}=\frac{f_{2}\left(x_{0}\right)}{\frac{f_{1}\left(x_{0}\right)}{f_{2}\left(x_{0}\right)}+1}=\frac{P_{2} f_{2}\left(x_{0}\right)}{\operatorname{Exp}\left[D\left(x_{0}\right)\right]+1} \\
P\left(R_{1} / x_{0}\right)=\frac{f_{1}\left(x_{0}\right)}{f_{1}\left(x_{0}\right)+f_{2}\left(x_{0}\right)}=\frac{f_{1}\left(x_{0}\right)}{\frac{f_{1}\left(x_{0}\right)}{f_{2}\left(x_{0}\right)}+1}=\frac{P_{1} f_{1}\left(x_{0}\right)}{\operatorname{Exp}\left[-D\left(x_{0}\right)\right]+1}
\end{gathered}
$$


Tenemos que: ecuación (23).

$$
P\left(R_{1} / x_{0}\right)>P\left(R_{2} / x_{0}\right)
$$

El vector aleatorio $x_{0}$ clasifica en la primera región $R_{1}$, de otra manera en $R_{2}$

\section{Metodología}

Para el desarrollo de esta investigación se utilizó un análisis cualitativo y cuantitativo, soportado en un análisis discriminante multivariado (ADM). A partir de los estados financieros suministrados por la Superintendencia de Industria y Comercio y las Cámaras de Comercio se calcularon los indicadores financieros de productividad y rentabilidad de las empresas del sector petróleo y gas en Colombia durante los periodos 2008 y 2010, con el fin de analizar si en los dos periodos señalados se puede evidenciar el mejoramiento de los indicadores de productividad y rentabilidad financiera señalados en los cuadros 1 y 2 del sector analizado.

Asimismo, como criterio de selección de variables que mejor discriminan en el análisis de la productividad y rentabilidad de las empresas del sector petróleo y gas en Colombia, se utilizó la distancia $\mathrm{D}^{2}$ de Mahalanobis.

\section{Población}

La población de esta investigación estuvo conformada por 116 empresas del sector petróleo y gas en Colombia que presentaron sus estados financieros entre 2008 y 2010.

\section{Fuentes y datos}

Se tomó como fuentes los estados financieros de la Superintendencia de Sociedades (2012), información registrada en la Cámara de Comercio de las empresas seleccionadas, asociada con los estados financieros de 2008 y 2010, de donde se tomaron los diferentes rubros financieros para calcular los indicadores de productividad y rentabilidad de las organizaciones de Colombia, labor que tomó seis meses de trabajo. 


\section{Análisis y discusión de los resultados}

Para analizar las variables asociadas a los indicadores seleccionados se utilizó el análisis discriminante por medio de software SPSS statistics 19, aplicación con la cual se establecieron las funciones discriminantes y se estudiaron los diferentes estadísticos; esta técnica permitió la estimación en un marco único para analizar si los indicadores financieros evaluados en el mismo contexto presentan diferencias significativas en los dos periodos seleccionados.

Seguidamente se verificó el cumplimiento de los supuestos requeridos para aplicar el análisis discriminante y, de esta forma, calcular y establecer la función objetivo con el fin de determinar qué indicadores discriminaban mejor para analizar la productividad y rentabilidad en las empresas del sector petróleo y gas en Colombia. También se calculó la precisión del modelo para predecir el comportamiento futuro de los indicadores en el sector. Por último, con los estadísticos, específicamente las medias de los indicadores para el sector, se analizó y evaluó el comportamiento de los diferentes indicadores financieros en los periodos 2008 y 2010.

\section{Verificación de los supuestos}

Para contrastar la normalidad de los datos se utilizó el estadístico del test de Shapiro y Wilk donde la hipótesis nula es la población que está distribuida normalmente. En esta prueba si el p-valor es mayor a alfa $(\alpha=0.05)$ no se rechaza la hipótesis y se concluye que los datos siguen una distribución normal. En el cuadro 3 se presenta los resultados de normalidad para los datos de los periodos 2008 y 2010, respectivamente, asociados a la prueba de Shapiro y Wilk. Las pruebas de normalidad para las variables por separado de los dos periodos respectivos dan como resultado que se comportan como variables normales, por lo que se verifica el supuesto de multinormalidad. 


\section{Cuadro3}

Prueba de Shapiro y Wilk para la comprobación de la normalidad de las razones financieras de 2008 y 2010 , respectivamente

\begin{tabular}{c|c|c|c|c}
\hline \multirow{2}{*}{ Indicador } & \multicolumn{4}{|c}{ Shapiro\&Wilk 2010 } \\
\cline { 2 - 5 } & Estadístico & gl & Estadístico & gl \\
\hline MB & 0.0374 & 116 & 0.00771 & 116 \\
\hline MO & 0.0261 & 116 & 0.048 & 116 \\
\hline MN & 0.0248 & 116 & 0.0444 & 116 \\
\hline IP1 & 0.0323 & 116 & 0.0186 & 116 \\
\hline IP2 & 0.0233 & 116 & 0.00508 & 116 \\
\hline IP3 & 0.0228 & 116 & 0.0406 & 116 \\
\hline IP4 & 0.00916 & 116 & 0.00857 & 116 \\
\hline IP5 & 0.00943 & 116 & 0.0393 & 116 \\
\hline IP6 & 0.00889 & 116 & 0.00604 & 116 \\
\hline
\end{tabular}

Homogeneidad de matrices de varianza-covarianza

La hipótesis nula de que no existen diferencias entre las matrices de varianza -covarianzas se probó con el test $\mathrm{M}$ de Box, el cual se basa en el cálculo de los determinantes de las matrices de covarianzas de cada grupo. El valor obtenido se aproxima por una F de Snedecor, si el p-valor es mayor que alfa $(\alpha=0.05)$ se acepta la igualdad entre las matrices de covarianzas. En el cuadro 4 se presentan los resultados del test M de box para 2008 y 2010.

Cuadro 4

\section{Resultados de la prueba de BOX}

\begin{tabular}{c|c|c}
\hline \multicolumn{2}{c|}{ M de Box } & 1493.601 \\
\hline \multirow{4}{*}{ F } & Aprox. & 31.82 \\
\cline { 2 - 3 } & gl1 & 45 \\
\cline { 2 - 3 } & g12 & 172251.496 \\
\cline { 2 - 3 } & Sig. & 0.237 \\
\hline
\end{tabular}

Los resultados obtenidos del estadístico de contraste $\mathrm{M}=1493.601 \mathrm{y}$ un valor de $\mathrm{F}=31.82$ con una probabilidad asociada $\mathrm{p}$ value $=0.237$ permiten aceptar con un 
buen grado de confianza la hipótesis nula de que no existen diferencias entre las matrices de covarianza de los dos grupos.

\section{Linealidad y multicolinealidad y singularidad}

Los supuestos de linealidad, multicolinealidad y singularidad no serán revisados, dado que para la construcción del modelo de análisis discriminante se utilizó el método por etapas o paso a paso, teniendo en cuenta el criterio de tolerancia para seleccionar las variables que son incluidas. De este modo, aquellas variables que presentan una correlación múltiple elevada con las restantes variables arrojarán una baja tolerancia y no serían consideradas de cara a la construcción de la función discriminante.

\section{Selección de las variables que mejor discriminan}

Como se mencionó anteriormente, el criterio de selección de las variables fue la distancia D2 de Mahalanobis. Se utilizaron todas las variables seleccionadas para el análisis de los dos periodos, constituyendo éstas las funciones discriminantes; el resultado final del modelo se muestra en el cuadro 5 y en las ecuaciones (24) y (25).

\section{Cuadro 5}

\section{Coeficiente de la función de clasificación}

\begin{tabular}{c|c|c}
\hline \multirow{2}{*}{ Indicador } & \multicolumn{2}{|c}{ Año } \\
\cline { 2 - 3 } & $\mathbf{2 0 0 8}$ & $\mathbf{2 0 1 0}$ \\
\hline MB & 2.471 & 2.834 \\
\hline MO & -1.788 & -2.5 \\
\hline MN & 12.167 & 10.912 \\
\hline IP1 & 1.89 & 1.297 \\
\hline IP2 & -3.323 & -3.57 \\
\hline IP3 & -7.9 & -5.703 \\
\hline IP4 & 1.668 & 1.548 \\
\hline IP5 & 2.62 & 2.52 \\
\hline IP6 & -4.244 & -4.582 \\
\hline (Constante) & -2.853 & -2.509 \\
\hline
\end{tabular}

Con base en los resultados, se calcularon las funciones discriminantes para pronosticar a futuro los diferentes indicadores de productividad y rentabilidad selec- 
cionados en las empresas del sector petróleo y gas en Colombia, ver ecuaciones (24) y (25).

$$
\begin{aligned}
& Z_{1}=\mathrm{MB} *(2.471)+\mathrm{MO} *(-1.788)+\mathrm{MN} *(12.167)+\mathrm{IP} 1 *(1.89)+\mathrm{IP} 2 *(-3.323)+\mathrm{IP} 3 * \\
& (-7.9)+\mathrm{IP} 4 *(1.668)+\mathrm{IP} 5 *(2.62)+\mathrm{IP} 6 *(-4.244)-\mathrm{K} *(2.853) \\
& Z_{2}=\mathrm{MB} *(2.834)+\mathrm{MO} *(-2.5)+\mathrm{MN} *(10.912)+\mathrm{IP} 1 *(1.297)+\mathrm{IP} 2 *(-3.57)+\mathrm{IP} 3 * \\
& (-5.703)+\mathrm{IP} 4 *(1.548)+\mathrm{IP} 5 *(2.52)+\mathrm{IP} 6(-4.582)-\mathrm{K} *(-2.509)
\end{aligned}
$$

Las magnitudes de los coeficientes de la función discriminante se pueden interpretar como indicadores de la importancia relativa de las variables en cada función discriminante. Así, si $\mathrm{a}_{\mathrm{ij}}$ es grande en valor absoluto, entonces hay una fuerte asociación entre la variable $\mathrm{Xj}$ (indicadores) y la función $\mathrm{Zi}$ (grupo), en relación con el resto de variables. Para el caso, se observa que los indicadores de margen neto (MN), utilidad neta/valor agregado (IP3) y utilidad neta/capital operativo (IP6) son los que tienen mayor importancia a la hora de definir el grupo de pertenencia.

En el cuadro 6 se muestran los resultados de la clasificación, la cual es en sí misma una validación de la función discriminante, puesto que expresa la capacidad predictiva de la función. De este modo, el 93\% de las empresas del grupo de 2008 son correctamente clasificadas y del segundo grupo (2010) en el $21.6 \%$ para una efectividad de clasificación promedio de la función de $57.3 \%$.

\section{Cuadro 6}

\section{Resultados de la clasificación}

\begin{tabular}{c|c|c|c|c|c}
\hline \multirow{2}{*}{} & \multirow{2}{*}{ Año } & \multicolumn{2}{|c|}{$\begin{array}{c}\text { Grupo de pertenencia } \\
\text { pronosticado (\%) }\end{array}$} & \multirow{2}{*}{ Total } \\
\cline { 3 - 5 } & & $\mathbf{2 0 0 8}$ & $\mathbf{2 0 1 0}$ & \\
\hline \multirow{3}{*}{ Original } & \multirow{3}{*}{ Recuento } & 2008 & 107 & 8 & 115 \\
\cline { 3 - 6 } & 2010 & 91 & 25 & 116 \\
\cline { 3 - 6 } & \multirow{2}{*}{$\%$} & 2008 & 93 & 7 & 100 \\
\cline { 3 - 6 } & & 2010 & 78.4 & 21.6 & 100 \\
\hline
\end{tabular}

Evaluación de la productividad y rentabilidad en el sector petróleo y gas en Colombia

$\mathrm{Al}$ analizar las funciones discriminantes generadas en el cuadro 5 se puede concluir que, en el análisis de la productividad y rentabilidad, los indicadores no presentan diferencias significativas en el transcurso de los años 2008 y 2010. De esta manera, cuando se revisan los estadísticos (Media) de los indicadores selecciona- 
dos de las empresas del sector petróleo y gas en Colombia, margen bruto (MB), margen operacional (MO), margen neto (MN), utilidad bruta/valor agregado (IP1), utilidad operacional/valor agregado (IP2), utilidad neta/valor agregado (IP3), valor agregado/capital operativo (IP4), utilidad operacional/capital operativo (IP5) y utilidad neta/ capital operativo (IP6), se observa que en el estudio de las medias los indicadores presentan valores cercanos en el transcurso del 2008 y 2010, como se muestra en el cuadro 7.

Cuadro 7

Medias de los indicadores financieros

\begin{tabular}{l|c|c|c}
\hline \multicolumn{2}{c|}{ Año } & Media & Desv. típ. \\
\hline \multirow{4}{*}{2008} & MB & 0.3129 & 0.68359 \\
\cline { 2 - 4 } & MO & 0.0013 & 0.78696 \\
\cline { 2 - 4 } & MN & -0.0699 & 0.82345 \\
\cline { 2 - 4 } & IP1 & 0.3192 & 1.02157 \\
\cline { 2 - 4 } & IP2 & -0.0186 & 0.9928 \\
\cline { 2 - 4 } & IP3 & -0.089 & 1.02296 \\
\cline { 2 - 4 } & IP4 & 1.7444 & 1.15419 \\
\cline { 2 - 4 } & IP5 & 0.174 & 0.21807 \\
\cline { 2 - 4 } & IP6 & 0.0785 & 0.18505 \\
\hline \multirow{5}{*}{2010} & MB & 0.2927 & 0.39252 \\
\cline { 2 - 4 } & MO & -0.0547 & 0.57808 \\
\cline { 2 - 4 } & MN & -0.0652 & 0.4969 \\
\cline { 2 - 4 } & IP1 & 0.1135 & 1.8644 \\
\cline { 2 - 4 } & IP2 & -0.0513 & 0.54717 \\
\cline { 2 - 4 } & IP3 & -0.1029 & 0.633 \\
\cline { 2 - 4 } & IP4 & 1.5888 & 1.15907 \\
\cline { 2 - 4 } & IP5 & 0.042 & 0.8194 \\
\cline { 2 - 4 } & IP6 & 0.023 & 0.39556 \\
\hline
\end{tabular}

Lo anterior demuestra que comparativamente el sector no mostró desarrollo y mejoramiento con respecto a los indicadores en estudio durante los periodos $2008 \mathrm{y}$ 2011, lo que muestra la necesidad de implementar estrategias que conduzcan al mejoramiento del sector.

Por otro lado, el estudio desarrollado por Suárez (2000) demuestra la efectividad de la metodología planteada por el análisis discriminante para la clasificación del nivel de productividad y rentabilidad de las empresas debido a la facilidad que éste 
establece para el manejo de múltiples variables. Asimismo, investigaciones como las desarrolladas por Fontalvo et al. (2011b) han demostrado que los procesos de estandarización con diferentes normas inciden positivamente en el mejoramiento de indicadores de las organizaciones en diferentes sectores empresariales; con esto se demuestra una estrategia de mejoramiento para el sector.

Es importante mencionar que en este artículo se presenta un método y un conjunto de herramientas que permiten analizar la productividad y rentabilidad de las empresas del sector petróleo y gas en Colombia; es por ello que Vivanco et al. (2010) hacen uso de la metodología que proporciona el análisis discriminante para determinar los niveles de competitividad de varias empresas de acuerdo con ciertas variables específicas. Esto demuestra la utilidad que tiene el ADM para la clasificación de empresas que presentan variables similares, pero las cuales es necesario diferenciarlas; además, se presenta una explicación clara de los indicadores seleccionados que mejoran de un periodo a otro, como lo señala Avendaño y Varela (2010), quienes afirman que la adopción de estándares puede afectar de manera positiva la competitividad y los indicadores financieros de la empresa o el sector que los implementa.

\section{Conclusiones}

Para esta investigación se utilizaron 116 empresas del sector petróleo y gas en Colombia; en esta muestra la precisión del modelo en el 2008 fue de $93 \%$ y en 2010 de $21.6 \%$, para un promedio total de clasificación del $57.3 \%$ de las empresas. De los indicadores, sólo el de margen bruto presentó una diferencia significativa en los dos periodos. El análisis de media del cuadro 7 muestra que $\mathrm{MB}=0.2927$; del año 2010 es menor con respecto al de 2008 (MB = 0.3129); lo anterior demuestra un deterioro en el margen logrado por las empresas después de haber cubierto los costos de los bienes vendidos.

Asimismo, con respecto a los indicadores margen operacional (MO), margen neto (MN), utilidad bruta/valor agregado (IP1), utilidad operacional/valor agregado (IP2), utilidad neta/valor agregado (IP3), éstos mostraron valores negativos en el 2010 , lo cual evidencia que en conjunto el costo de ventas, los gastos financieros incurridos y los impuestos superan el nivel de ingresos. De esta forma, los resultados indican que el sector de petróleo y gas en Colombia, durante los periodos evaluados 2008 y 2010, no tuvo mejoramiento en sus indicadores persistiendo condiciones críticas en los resultados del sector. 
Por otra parte, esta investigación permitió establecer una función objetiva para las empresas evaluadas en el sector petróleo y gas en Colombia, con lo que se puede estudiar y analizar qué indicadores discriminan mejor y así poder tomar acciones teniendo en cuenta el cálculo y estudio de los indicadores de productividad que presenten una buena discriminación.

Es importante señalar que en investigaciones previas de Fontalvo et al. (2012a, 2012b, 2011a y 2011b), se ha demostrado la efectividad de la aplicación metodológica del análisis discriminante en el comportamiento de indicadores financieros en otros sectores con lo que se evidencia la pertinencia de la metodología desarrollada.

A partir de estas evidencias se podría contribuir al desarrollo de estudios similares en otros sectores, considerando siempre el marco de articulación entre las variables que afectan a la organización misma, dependiendo de su sector, así como a los intereses institucionales de los actores intervinientes que se derivan de la separación de la propiedad y el control organizacional (Vargas, 2005), lo que muy probablemente podría modificar los indicadores de desempeño y contribuir en el desarrollo de políticas empresariales y la toma de decisiones estratégicas, las cuales lleven a la organización a niveles más competitivos, sin descuidar lo concerniente a su responsabilidad social.

\section{Referencias}

Altahyde, J. (2012). A politica energética brasileira na era da globalização: energia e conflitos de um estado emdesenvolvimento. Revista de Sociologia e Política 20 (41): 75-91.

Agencia Nacional de Hidrocarburos (ANH) (2007). Ministerio de Minas y Energías. Disponible en: http://www.anh.gov.co/ronda2007.anh.gov.co/internae8ec.html?id=91

Área de Investigaciones Económicas de Correval (2012). Colombia: situación actual y perspectivas económicas. Disponible en: https://www.correval.com/ InvEcon/index.php/component/content/frontpage 
Avendaño, B. y R. Varela (2010). La adopción de estándares en el sector hortícola de Baja California. Estudios Fronterizos 11 (1): 171-202.

Banco Nacional de Comercio Exterior de México (1991). La productividad y la capacitación, Pilares de la Modernización. Revista de Comercio Exterior 41 (1): 20-32.

Berechet, C. y F. San Miguel (2006). Innovación y productividad en la economía de Navarra. Posicionamiento frente a la regiones europeas más avanzadas. Centro para la Competitividad de Navarra 4 (2): 14-25.

Brealey, R. y S. Myers (1998). Fundamentación de financiación empresarial. 5a. ed., Madrid: McGraw-Hill.

Buenaventura, G. (2002). El estudio de apalancamientos como metodología de análisis de la gestión en la empresa. Estudios Gerenciales 82: 65-91. Disponible en: http://www.redalyc.org/articulo.oa?id=21208204

Carvajal, P. et al. (2004). Aplicación del análisis discriminante para explorar la relación entre el examen de ICFES y el rendimiento en álgebra lineal de los estudiantes de ingeniería de la UTP en el periodo 2001-2003. Scientia et Technica 12 (4): 28-58.

Departamento Administrativo Nacional de Estadística (DANE) (2011). Colombia, exportaciones de café, carbón, petróleo y sus derivados, ferroníquel y no tradicionales. Disponible en: https://www.dane.gov.co/index.php/comercio-y-servicios/comercio-exterior/exportaciones

Díaz, N. (2009). Ranking financiero: Herramienta financiera para medir la productividad de las empresas de comercialización de energía eléctrica. Revista Estudios Gerenciales 25 (111): 13-34. Disponible en: http://www.redalyc. org/articulo.oa?id=21211972002.

Elizondo, A. y E. Altman (2003). Medición integral del riesgo de crédito. México: Limusa. 
Fontalvo, T. et al. (2011a). Evaluación del impacto de los sistemas de gestión de la calidad en la liquidez y rentabilidad de las empresas de la zona industrial de Mamonal (Cartagena, Colombia). Revista Católica del Norte, septiembre-diciembre (34): 1-28.

(2011b). Aplicación del análisis discriminante para evaluar el mejoramiento de los indicadores financieros en las empresas del sector extracción de petróleo crudo y gas natural en Colombia. Revista Soluciones de Postgrado EIA, julio-diciembre (7): 1-16.

(2012a). Evaluación del impacto de los sistemas de gestión de la calidad en la liquidez y rentabilidad de las empresas de la zona industrial Vía 40. Revista Pensamiento y Gestión 32 (1): 3-25.

(2012b). Aplicación de análisis discriminante para evaluar el mejoramiento de los indicadores financieros en las empresas del sector alimento de Barranquilla, Colombia. Ingeniare Revista Chilena de Ingeniería 20 (3): 320-330.

Giner Inchausti, B. (1990). Información contable y toma de decisiones. Revista Española de financiación y contabilidad 20 (62): 27-43.

Guajardo, G. (2002). Contabilidad financiera, México: McGraw-Hill. 539 pp.

Lachenbruch, P. (1975). Discriminant analysis. Nueva York: Macmillan Pub Co.: 250.

Levy, L. (2004). Planeación financiera en la empresa moderna: el mejoramiento estratégico de las finanzas para lograr el éxito empresarial. México: ISEF 20 (4): 35-50.

López, E. et al. (2012). La economía petrolera en Colombia: marco legal-contractual y sus principales efectos sobre la actividad económica del país (parte I). Banco de La República. Bogotá, Colombia 12 (692): 25-46.

Milind, L. (1995). Desarrollo del apalancamiento estratégico. Madrid: Ediciones Díaz de Santos. 
Ministerio de Relaciones Exteriores (2011). Colombia, un país en diversidad energética. República de Colombia. Fecha de consulta: marzo 13 de 2013 Disponible en: http://www.cancilleria.gov.co/sites/default/files/DocEstrategicos/EnergiaWeb.pdf

Miranda, J. y L. Toirac (2010). Indicadores de productividad para la industria dominicana, Revista Ciencia y Sociedad 35 (2): 235-290. Disponible en: http:// www.redalyc.org/articulo.oa?id=87014563005.

Mures, J. et al. (2005). Aplicación del análisis discriminante y regresión logística en el estudio de la morosidad en las entidades financieras. Comparación de resultados. Pecvnia 1 (1): 175-199.

Navas, R. y A. Marbelis (2009). Análisis financiero: una herramienta clave para una gestión financiera eficiente. Revista Venezolana de Gerencia 14 (48): 606- 628 .

Nuñez, A. y R. Vieites (2009). El diagnóstico financiero de la empresa. CEEI Galicia 12 (2): 5-32.

Ortiz, H. (2011). Análisis financiero aplicado y principios de administración financiera. 14a. ed., Colombia: Universidad Externado de Colombia.: 118.

Palacio, J. (2008). Importancia del diagnóstico económico financiero para conocer su posición económica financiera de la empresa. Revista Altair Consultores 14 (2): 5-20.

Rodríguez, P. (2004). Petróleo y tercermundismo. Compendium 6 (12): 59-70. Disponible en: http://www.redalyc.org/articulo.oa?id=88001205

Selling, T. y C. Stickney (1989). The effects of business environment and strategy on a firm's rate of return on assets. Financial Analysts Journal 45 (1): 43-52.

Stickney, C.P. (1990) Financial Statement Analysis A Strategic Perspective. San Diego, California: Harcourt Brace Jovanovich Publisher. 
Suárez, J. (2000). Los parámetros característicos de las empresas manufactureras de alta rentabilidad: una aplicación del análisis discriminante. Revista Española de Financiación y Contabilidad 29 (1): 443-481.

Superintendencia de Sociedades (2012). SIREM. Disponible en: http://sirem.supersociedades.gov.co/SIREM/.

Tabachnick, B. y L. Fidell (2001). Using multivariate statistics. 4ª edición Boston: Pearson.

Vallejo, G. (2012). Análisis multivariantes aplicados a las ciencias comportamentales. Oviedo: Servicio de Publicaciones de la Universidad de Oviedo.

Vargas, J. (2005). Análisis de fundamentos de la teoría institucional. Revista Digital Universitaria 6 (8): 2-21. Disponible en: http://www.revista.unam.mx/vol.6/ num8/art84/int84.htm

Vázquez, J. (2001). Resultados del actual proceso de cambio en las estructuras de activo y de pasivo empresarial: La complementariedad e interdependencia entre el apalancamiento operativo y el apalancamiento financiero. Investigaciones Europeas de Dirección y Economía de la Empresa 7 (3): 67-90.

Villamil, J. (2007). Aproximación a los recursos minero-energéticos nacionales y el capital extranjero en Colombia. Revista Gestión y Ambiente 10 (2): 61-72.

Vivanco, M. (2010). Análisis de competitividad de cuatro sistemas-productos estatales de tilapia en México. Revista Estudios Sociales 18 (1): 165-207. Disponible en: http://www.redalyc.org/articulo.oa?id=41712074005. 
